\title{
Relativistic Atomic Calculations of Spectral Parameters of Be-Like Tungsten Ion
}

\author{
K. HAMASHA \\ Department of Basic Sciences, Al-Huson University College,
} Al-Balqa Applied University, Al-Salt 19117, Jordan

Received: 27.11.2020 \& Accepted: 12.01.2021

Doi: $10.12693 /$ APhysPolA.139.151

*e-mail: khamasha@bau.edu.jo

\begin{abstract}
Comprehensive large scale atomic spectral data calculations were performed for a Be-like tungsten ion $\left(\mathrm{W}^{70+}\right)$, starting from a shell number $n=2$, through $n=3,4,5,6,7$, and ending at a shell number $n=8$. We applied the relativistic configuration interaction method of the flexible atomic code. The generated data include detailed information concerning allowed and forbidden transitions, together with transition energies, transition wavelengths, transition rates and oscillator strengths for electric-dipole (E1), electric-quadruple (E2), electric-octupole (E3), magnetic-dipole (M1), magneticquadruple (M2) and magnetic-octupole (M3) transitions. The produced atomic data are satisfactory when compared to the previously published results calculated by GRASP2K code. The procured multipole transitions data were used to construct synthetic spectra for allowed and forbidden transitions of $\mathrm{W}^{70+}$. We simulated the experimental spectra of low-density $\mathrm{W}$ plasma and found the spectral range $0.6-1.4 \AA$. The produced atomic data is important for several research branches, including fusion research and plasma physics.
\end{abstract}

topics: electric dipole transitions, forbidden transitions, oscillator strengths, transition rates

\section{Introduction}

Accurate atomic structure and spectral data of highly ionized tungsten ions play an important role in plasma science, high energy astrophysics and fusion reaction research. Tungsten atoms are used as a basic construction material in tokamak plasma devices. Consequently, they appear as impurities introduced by subsidiary erosion of probe surfaces. The presence of highly ionized tungsten ions, even in small amounts, affects the achieved temperature and the confinement times in tokamak devices. Therefore, many ionization stages of experimental plasma spectra may include numerous spectral lines linked to multiple ionization stages of tungsten ions. In order to diagnose plasma by analyzing the radiation emission profile of fusion plasma, and further to interpret experimental spectra of tungsten ions, it is necessary to have theoretical atomic data for most ionizing stages of tungsten ions. Such data can be used to construct the theoretical spectra for a wide temperature range and various ionization stages of $\mathrm{W}[1,2]$.

Data of spectral lines of highly ionized ions reveal atomic structure information that expedites the testing of atomic theories. This includes the relativistic quantum electrodynamics corrections (QED) and the correlation effects. The intensities and profiles of spectral lines yield diagnostic information of the plasma state. This, in fact, justifies an immense effort to collect experimental and theoretical atomic data for tungsten ions [3-17]. The experimental facility that is widely used in studying the spectra of tungsten ions is the electron beam ion trap (EBIT). With it, one produces spectra of highly charged ions under well-controlled conditions [13-17]. The accuracy of collected data has improved significantly due to the enhanced measurements achieved by studying the X-ray spectra of laser-produced plasmas [8-12].

There are a few computational methods and various atomic codes modelling the experimental spectra of tungsten or allowing to perform pure theoretical calculations for particular tungsten ions. For instance, HULLAC code allows to produce high quality atomic data for tungsten ions. The code is based on the multi-configuration Dirac-Fock (MCDF) method and gives a good agreement with experiment [18]. Similarly, the flexible atomic code (FAC) demonstrates excellent capabilities in producing accurate atomic data. The FAC bases on the MCDF method with modified approximations and gives results consistent with the experimental data [19]. Several approximations have been used in previous studies in order to improve the quality and accuracy of the theoretical atomic data of tungsten ions [20-25]. Singh et al. published theoretical results of the energy levels and radiative properties of Be-like W obtained using the MCDHF method implemented in GRASP2K code [26]. The calculations 
include the energy levels obtained by the FAC for a comparison. It turned out that the comparison between the calculated data was not comprehensive because they were limited to multipole transitions from the ground state only. In the present paper, the relativistic configuration interaction (RCI) method of the FAC is used to carry out wideranging and large-scale atomic calculations of allowed and forbidden transitions of a Be-like tungsten ion $\left(\mathrm{W}^{70+}\right)$ from a shell number $n=2$ to a shell number $n=8$. The main goal of this paper is to produce accurate and comprehensive atomic data that will provide direct access to important spectroscopic properties of $\mathrm{W}^{70+}$. Our findings will be tested and benchmarked with the atomic theories and computational methods of the FAC and GRASP2K published in [26]. The obtained data includes transition energies, oscillator strengths and transition rates for allowed transitions (electric dipole E1) and forbidden (multipole) transitions, electricquadruple E2, electric-octupole E3, magneticdipole M1, magnetic-quadruple M2 and magneticoctupole M3 transitions. The produced atomic data are then used to construct theoretical atomic spectra for allowed and forbidden transitions of $\mathrm{W}^{70+}$. Note that the atomic structure and spectral properties are essential for the understanding and diagnosing of ion/atom-electron collision processes in plasma [27, 28].

\section{Theoretical formulation}

A brief explanation of the used method (the RCI method) is presented in this section. For the detailed formulation of this method we refer to the original paper of the FAC [19].

In general, calculations start from the Dirac equations. The ground state configuration for a Be-like $\mathrm{W}$ ion is used to construct a mean configuration with a fractional occupation number which takes into account the electron screening of the configurations involved. A modified self-consistent DiracFock-Slater iteration is performed in order to derive a local central potential that is used to derive the radial orbitals for the construction of basis states. The radial orbital from the previous step is used to derive the potential. The Dirac coupled equations are converted to a Schrödingerlike equation by eliminating a small component and performing some appropriate transformations. We used the standard Numerov method to solve the Schrödinger-like equation. Energy levels are determined by diagonalizing the Hamiltonian. To reduce errors in energy levels, a correction procedure is used. The electron-electron interaction includes the spherically averaged potential due to bound electrons and local approximations to the exchange interactions. Therefore, the asymptotic behavior at large $r$ is corrected. The radial function, which is not linear, covers a large radial distance. The minimum and maximum distances on the radial grid are chosen to be within the nuclear charge distribution and the excited states to be below the shell number 20. The bound energies are less than the Coulomb potential at $r_{\max }$. Radiative transition rates are calculated in a single multipole approximation, where the initial $(i)$ state is $\psi_{i}=\sum_{v} b_{i v} \varphi_{v}$, the final $(f)$ state is given as $\psi_{f}=\sum_{\mu} b_{f \mu} \varphi_{v \mu}$ and the multipole operator is $O_{M}^{L}$. The second quantization method is used to solve the Hamiltonian matrix elements by recoupling the creation and annihilation operators with the help of the Racah algebra. The generalized line strength of the transition is $S_{f i}=\left|\left\langle\psi_{f}\left\|O_{M}^{L}\right\| \psi_{i}\right\rangle\right|^{2}$ and the weighted oscillator strength is given by

$$
g f_{f i}=[L]^{-1} \hbar \omega(\alpha \hbar \omega)^{2 L-2} S_{f i} .
$$

Finally, the weighted radiative transition rate (in atomic units a.u.) is given by

$$
g_{f} A_{f i}=\frac{1}{2}(\hbar \omega)^{2} \alpha^{3} g_{i} f_{i f},
$$

where $\hbar \omega=E_{f}-E_{i}$ is the transition energy.

\section{Results and discussion}

The calculations started from the ground state electronic configurations towards configurations related to a single excitation for $\mathrm{W}^{70+}$. In the ground electronic configuration of the Be-like $\mathrm{W}$, there are two valance electrons. One can write then $1 s^{2} 2 s^{2}$ for the shell number $n=1$. This means that its ground state is ${ }^{1} S_{0}$. The calculations follow the RCI method discussed in the theoretical formulation section.

The electronic configurations which are included for the shell number $n=2$ are $1 s^{2} 2 s^{2}, 1 s^{2} 2 p^{2}$, $1 s^{2} 2 s 2 p$.

In the case of the shell number $n=3$, electronic configurations include: $1 s^{2} 2 s 3 s, 1 s^{2} 2 s 3 p, 1 s^{2} 2 p 3 p$, $1 s^{2} 2 s 3 d, 1 s^{2} 3 s^{2}, 1 s^{2} 3 p^{2}, 1 s^{2} 3 d^{2}, 1 s^{2} 2 p 3 d, 1 s^{2} 2 p 3 s$, $1 s^{2} 3 p 3 d, 1 s^{2} 3 p 3 s$.

Further, for $n=4$, one has: $1 s^{2} 2 s 4 s, 1 s^{2} 2 s 4 p$, $1 s^{2} 2 s 4 d, \quad 1 s^{2} 2 s 4 f, \quad 1 s^{2} 2 p 4 p, 1 s^{2} 2 p 4 d, 1 s^{2} 2 p 4 f$, $1 s^{2} 4 s^{2}, 1 s^{2} 4 p^{2}, 1 s^{2} 4 d^{2}, 1 s^{2} 4 f^{2}$.

For $n=5$, one has: $1 s^{2} 2 s 5 s, 1 s^{2} 2 s 5 p, 1 s^{2} 2 s 5 d$, $1 s^{2} 2 s 5 f, \quad 1 s^{2} 2 s 5 g, 1 s^{2} 2 p 5 p, 1 s^{2} 2 p 5 d, 1 s^{2} 2 p 5 f$, $1 s^{2} 2 p 5 g, 1 s^{2} 5 s^{2}, 1 s^{2} 5 p^{2}, 1 s^{2} 5 d^{2}, 1 s^{2} 5 f^{2}, 1 s^{2} 5 f^{2}$, $1 s^{2} 2 p 5 s$.

For $n=6$, one has: $1 s^{2} 2 s 6 s, 1 s^{2} 2 s 6 p, 1 s^{2} 2 s 6 d$, $1 s^{2} 2 s 6 f, \quad 1 s^{2} 2 s 6 g, \quad 1 s^{2} 2 p 6 p, \quad 1 s^{2} 2 p 6 d, 1 s^{2} 2 p 6 f$, $1 s^{2} 2 p 6 g, 1 s^{2} 6 s^{2}, 1 s^{2} 6 p^{2}, 1 s^{2} 6 d^{2}, 1 s^{2} 6 f^{2}, 1 s^{2} 6 f^{2}$.

For $n=7$, one has: $1 s^{2} 2 s 7 s, 1 s^{2} 2 s 7 p, 1 s^{2} 2 s 7 d$, $1 s^{2} 2 s 7 f, \quad 1 s^{2} 2 s 7 g, \quad 1 s^{2} 2 p 7 p, \quad 1 s^{2} 2 p 7 d, 1 s^{2} 2 p 7 f$, $1 s^{2} 2 p 7 g, 1 s^{2} 7 s^{2}, 1 s^{2} 7 p^{2}, 1 s^{2} 7 d^{2}, 1 s^{2} 7 f^{2}, 1 s^{2} 7 f^{2}$.

And for $n=8$, one has: $1 s^{2} 2 s 8 s, 1 s^{2} 2 s 8 p$, $1 s^{2} 2 s 8 d, \quad 1 s^{2} 2 s 8 f, \quad 1 s^{2} 2 s 8 g, \quad 1 s^{2} 2 p 8 p, \quad 1 s^{2} 2 p 8 d$, $1 s^{2} 2 p 8 f, 1 s^{2} 2 p 8 g, 1 s^{2} 8 s^{2}, 1 s^{2} 8 p^{2}, 1 s^{2} 8 d^{2}, 1 s^{2} 8 f^{2}$, $1 s^{2} 8 f^{2}$.

The calculations for $n=2,3,4,5,6,7$ and 8 yield 474 energy states of even and odd parities. In the case of the electron which is excited, its electronic configurations included in the calculation are 
denoted as $2 s^{2}, 2 s^{1} n l, 2 p n l$ where $n=2,3,4,5$, 6,7 and 8 , and $l$ spans all the allowed orbital angular momenta for a given $n$. As a reminder, the first electron in the considered system is always in the ground state. Now, the inner shell transitions of $n=2$, which are expected to be in the UV range, are not included. The effect of high $n$-shell numbers in the configuration interaction is very small. The most important configurations are those of $n=2$, 3 and $n=4$. Details are presented in the next sections.

\section{Allowed transitions}

A large number of optically allowed transitions is produced but only strong transitions have significant values. A filtration procedure based on the spectral ranges of the data is applied, where only transition lines of the highest values of the radiative transition probability $\left(A_{r}\right)$ and the oscillator strength $\left(f_{i j}\right)$ are considered. The strong transition lines are collected in Table I. One can depicted the characteristic types of transitions of M1, namely, $n p-2 s, n s-2 p$ and $n d-2 p$ (the most dominant).

In general, the strongest transition is $3 d_{5 / 2} \rightarrow 2 p_{3 / 2}$, with radiative transitions probability equal to $1.6974 \times 10^{+15} \mathrm{~s}^{-1}$ at wavelength $\lambda=1.2520 \AA$. In the case of $n p-2 s$ group, the strongest transition line is $3 p_{1 / 2}-2 s_{1 / 2}$, with radiative transition probability equal to $2.4306 \times 10^{+14} \mathrm{~s}^{-1}$ at wavelength $\lambda=1.3368 \AA$. In the third group $(n s \rightarrow 2 p)$, one has mainly weak transitions. Blending among transition lines makes it difficult to identify the exact transition, thus a line identification is assigned to the strongest transition. For example, in Table I, there are two blended transitions with wavelengths $0.7590 \AA$ and $0.7591 \AA$ related to the transitions $5 d_{3 / 2} / J=0^{-}$ $2 p_{1 / 2} / J=1$ and $5 d_{3 / 2} / J_{=1}-2 p_{1 / 2} / J=1$, respectively. In another example, the two transitions with wavelengths $0.9336 \AA$ and $0.9338 \AA$ are related to $5 g_{7 / 2} / J=3^{-}-2 p_{3 / 2} / J_{=2}$ and $5 d_{3 / 2} / J_{=2}-2 p_{3 / 2} / J=1$, respectively.

The produced atomic data is compared with data published in [26], calculated by using the relativistic GRASP2K code (Table I), which is discussed in detail in $[29,30]$. It is worth noting that the atomic data published in [26] does not include all possible strong transitions for radiative properties - they are limited to the transitions to the ground state only. Weak transitions, which are not expected to appear in a normal tungsten spectrum, are also presented [26]. Findings reported in the current paper include important transitions that are expected to appear in the experimental tungsten spectra. Nevertheless, there are good agreements in the atomic data that correspond to wavelengths, $A$-values and oscillator strengths of E1 transitions. The maximum percentage difference in the values of the wavelengths is about $0.409 \%$ and the maximum percentage in the oscillator strength is about $0.927 \%$.
A comparison of the energy levels of the Be-like W obtained by using the FAC and GRASP2K programs is presented by Singh et al. [26], and demonstrates good agreement. Thus, calculations of energy levels are not presented in this paper in order to avoid repetition. GRASP2K and the FAC include all correlation effects by following the multiconfiguration interaction but the electron-electron interaction potential has different approximations. The finite nuclear potential in the FAC is assumed to be the potential of a uniform charged ball of a statistical model radius but the Thomas-Fermi nuclear charge distribution form is the nuclear potential used in GRASP2K.

The main effective difference in the two codes is: the FAC includes the lowest order one-electron QED effects (vacuum polarization and self-energy) but does not include the Breit corrections, while in GRASP2K, both kinds of corrections are included. The correlation effects in highly charged ions are important as the QED effects but the Breit interaction is the most important missing term.

\section{Forbidden transitions}

Forbidden transitions play the main role in plasma diagnostics because the presence of forbidden transition lines in experimental spectra yields information about electron density and temperature. According to the multipole selection rules, the electric dipole transitions are much stronger than the other multipole transitions.

The E1 transitions are considered optically allowed transitions, while the other weak transitions are considered forbidden transitions. The calculated data for forbidden transitions include the electric-quadrupole E2, electric-octupole E3, magnetic-dipole M1, magnetic-quadrupole M2 and magnetic-octupole M3 transitions.

The calculated atomic data of the most important transitions of electric quadrupole E2 of the Be-like tungsten ion are shown in Table SI in supplementary material [31]. Among all the collected transitions, the $n p-2 p$ type transition dominates. The strongest transition is: $3 p_{1 / 2}-2 p^{3 / 2}$ with radiative transition probability of $3.365 \times 10^{+12}(\mathrm{~s}-1)$ at $\lambda=1.3173 \AA$. The transitions are grouped as: $n s-2 p, n p-2 p$ and $n g-2 p$. The intensity ratio of radiative E2 transitions compared to E1 transitions $(\mathrm{E} 2 / \mathrm{E} 1)$ are about $10^{-4}$.

The dominant transitions for electric-octupole (E3) belong to the $n d-2 p$ type. Among this type, the strongest transition is $3 d_{3 / 2}-2 p_{3 / 2}$, with radiative transition probability of $1.521 \times 10^{+10} \mathrm{~s}^{-1}$ at $\lambda=1.2545 \AA$. The other groups of E3 transitions are: $n g-2 p$ and $n f-2 p$. The most important transitions are listed in Table SII [31] The intensity ratio of E3 transitions compared to E1 transitions $(\mathrm{E} 3 / \mathrm{E} 1)$ is about $10^{-5}$. 
TABLE I

Transition energy $(\Delta E[\mathrm{eV}])$, wavelength $(\lambda[\AA])$, radiative transition rates $\left(A_{r}\left[\mathrm{~s}^{-1}\right]\right)$ and weighted oscillator strengths $\left(g f_{i j}\right)$ for strong electric dipole transitions (E1) of Be-like tungsten ion calculated by RCI method of FAC.

\begin{tabular}{|c|c|c|c|c|c|c|c|c|c|}
\hline$P_{\text {up }}$ & Upper state & $J_{\text {up }}$ & $P_{\text {low }}$ & Lower state & $J_{\text {low }}$ & $\Delta E[\mathrm{eV}]$ & $g f_{i j}$ & $A_{r}\left[\mathrm{~s}^{-1}\right]$ & $\lambda[\AA]$ \\
\hline \multirow[t]{2}{*}{1} & $2 s_{1 / 2} 7 p_{3 / 2}$ & 1 & 0 & $2 s_{1 / 2}^{2}$ & 0 & 17412.60 & 0.03236 & $1.34 \times 10^{14}$ & 0.7120 \\
\hline & & & & & & & $0.0311^{a}$ & $1.37 \times 10^{14 a}$ & $0.7117^{a}$ \\
\hline \multirow[t]{2}{*}{1} & $2 p_{1 / 2} 7 i_{11 / 2}$ & 0 & 1 & $2 s_{1 / 2} 2 p_{3 / 2}$ & 1 & 17369.79 & 0.0163 & $7.65 \times 10^{13}$ & 0.7138 \\
\hline & & & & & & & $0.0177^{a}$ & $7.74 \times 10^{13 a}$ & $0.7138^{a}$ \\
\hline 0 & $2 s_{1 / 2} 6 d_{3 / 2}$ & 2 & 1 & $2 s_{1 / 2} 2 p_{1 / 2}$ & 1 & 17210.68 & 0.0624 & $1.60 \times 10^{14}$ & 0.7204 \\
\hline 1 & $2 p_{3 / 2} 6 d_{3 / 2}$ & 0 & 0 & $2 p_{1 / 2} 2 p_{3 / 2}$ & 1 & 17192.73 & 0.0128 & $1.64 \times 10^{14}$ & 0.7211 \\
\hline 1 & $2 p_{3 / 2} 6 d_{3 / 2}$ & 3 & 0 & $2 p_{1 / 2} 2 p_{3 / 2}$ & 2 & 17174.08 & 0.0879 & $1.61 \times 10^{14}$ & 0.7219 \\
\hline 1 & $2 p_{1 / 2} 6 d_{3 / 2}$ & 1 & 0 & $2 p_{1 / 2}^{2}$ & 0 & 17125.74 & 0.0519 & $2.20 \times 10^{14}$ & 0.7239 \\
\hline \multirow[t]{2}{*}{0} & $2 p_{1 / 2} 2 p_{3 / 2}$ & 1 & 0 & $2 s_{1 / 2}^{2}$ & 0 & 16549.86 & 0.0481 & $1.92 \times 10^{13}$ & 0.7491 \\
\hline & & & & & & & $0.0503^{a}$ & $2.00 \times 10^{13 a}$ & $0.7489^{a}$ \\
\hline \multirow[t]{2}{*}{0} & $2 p_{1 / 2} 5 p_{1 / 2}$ & 0 & 1 & $2 s_{1 / 2} 2 p_{1 / 2}$ & 1 & 16450.62 & 0.0051 & $1.71 \times 10^{13}$ & 0.7536 \\
\hline & & & & & & & $0.0041^{a}$ & $1.63 \times 10^{13 a}$ & $0.7537^{a}$ \\
\hline 0 & $2 p_{3 / 2}^{2}$ & 1 & 1 & $2 s_{1 / 2} 2 p_{1 / 2}$ & 0 & 16383.37 & 0.0507 & $1.97 \times 10^{14}$ & 0.7567 \\
\hline 0 & $2 p_{3 / 2}^{2}$ & 2 & 1 & $2 s_{1 / 2} 2 p_{1 / 2}$ & 1 & 16350.44 & 0.1199 & $2.78 \times 10^{14}$ & 0.7583 \\
\hline 1 & $2 p_{3 / 2} 5 \mathrm{~d}_{3 / 2}$ & 0 & 0 & $2 p_{1 / 2} 2 p_{3 / 2}$ & 1 & 16334.19 & 0.0244 & $2.83 \times 10^{14}$ & 0.7590 \\
\hline 1 & $2 p_{3 / 2} 5 \mathrm{~d}_{3 / 2}$ & 1 & 0 & $2 p_{1 / 2} 2 p_{3 / 2}$ & 1 & 16333.23 & 0.0607 & $2.34 \times 10^{14}$ & 0.7591 \\
\hline 1 & $2 p_{3 / 2} 5 d_{3 / 2}$ & 3 & 0 & $2 p_{1 / 2} 2 p_{3 / 2}$ & 2 & 16314.13 & 0.1678 & $2.77 \times 10^{14}$ & 0.7600 \\
\hline 1 & $2 p_{1 / 2} 5 d_{3 / 2}$ & 1 & 0 & $2 p_{1 / 2}^{2}$ & 0 & 16267.08 & 0.0978 & $3.74 \times 10^{14}$ & 0.7622 \\
\hline 1 & $2 p_{3 / 2} 7 d_{5 / 2}$ & 3 & 0 & $2 p_{3 / 2}^{2}$ & 2 & 16226.61 & 0.0941 & $1.54 \times 10^{14}$ & 0.7641 \\
\hline 0 & $2 s_{1 / 2} 6 d_{5 / 2}$ & 3 & 1 & $2 s_{1 / 2} 2 p_{3 / 2}$ & 2 & 15778.96 & 0.0967 & $1.49 \times 10^{14}$ & 0.7857 \\
\hline 1 & $2 p_{3 / 2} 6 d_{5 / 2}$ & 3 & 0 & $2 p_{3 / 2}^{2}$ & 2 & 15715.12 & 0.1595 & $2.44 \times 10^{14}$ & 0.7889 \\
\hline 1 & $2 p_{1 / 2} 6 d_{5 / 2}$ & 3 & 0 & $2 p_{1 / 2} 2 p_{3 / 2}$ & 2 & 15685.89 & 0.0991 & $1.51 \times 10^{14}$ & 0.7904 \\
\hline 1 & $2 p_{3 / 2} 6 d_{5 / 2}$ & 1 & 0 & $2 p_{3 / 2}^{2}$ & 0 & 15646.93 & 0.0440 & $1.56 \times 10^{14}$ & 0.7924 \\
\hline 0 & $2 p_{3 / 2} 4 p_{3 / 2}$ & 3 & 1 & $2 s_{1 / 2} 2 p_{3 / 2}$ & 2 & 14996.68 & 0.1686 & $2.35 \times 10^{14}$ & 0.8267 \\
\hline 0 & $2 p_{1 / 2} 4 p_{3 / 2}$ & 1 & 1 & $2 s_{1 / 2} 2 p_{1 / 2}$ & 0 & 14980.78 & 0.0465 & $1.51 \times 10^{14}$ & 0.8276 \\
\hline \multirow[t]{2}{*}{1} & $2 s_{1 / 2} 4 p_{3 / 2}$ & 1 & 0 & $2 s_{1 / 2}^{2}$ & 0 & 14962.10 & 0.0787 & $2.52 \times 10^{14}$ & 0.8286 \\
\hline & & & & & & & $0.0831^{a}$ & $2.69 \times 10^{14 a}$ & $0.8284^{a}$ \\
\hline \multirow[t]{2}{*}{0} & $2 p_{1 / 2} 4 p_{3 / 2}$ & 2 & 1 & $2 s_{1 / 2} 2 p_{1 / 2}$ & 1 & 14947.17 & 0.0283 & $8.09 \times 10^{13}$ & 0.8295 \\
\hline & & & & & & & $0.0243^{a}$ & $7.59 \times 10^{13 a}$ & $0.8298^{a}$ \\
\hline 0 & $2 s_{1 / 2} 5 d_{5 / 2}$ & 3 & 1 & $2 s_{1 / 2} 2 p_{3 / 2}$ & 2 & 14929.15 & 0.1933 & $2.67 \times 10^{14}$ & 0.8305 \\
\hline 0 & $2 p_{3 / 2} 4 p_{3 / 2}$ & 0 & 1 & $2 s_{1 / 2} 2 p_{3 / 2}$ & 1 & 14926.05 & 0.0220 & $2.13 \times 10^{14}$ & 0.8306 \\
\hline 0 & $2 p_{3 / 2} 4 p_{3 / 2}$ & 1 & 1 & $2 s_{1 / 2} 2 p_{3 / 2}$ & 1 & 14905.70 & 0.0646 & $2.08 \times 10^{14}$ & 0.8318 \\
\hline 1 & $2 p_{3 / 2} 5 d_{5 / 2}$ & 3 & 0 & $2 p_{3 / 2}^{2}$ & 2 & 14866.91 & 0.3177 & $4.35 \times 10^{14}$ & 0.8339 \\
\hline 1 & $2 p_{3 / 2} 5 d_{5 / 2}$ & 2 & 0 & $2 p_{3 / 2}^{2}$ & 2 & 14864.24 & 0.1008 & $1.93 \times 10^{14}$ & 0.8341 \\
\hline 1 & $2 p_{1 / 2} 5 d_{5 / 2}$ & 2 & 0 & $2 p_{1 / 2} 2 p_{3 / 2}$ & 1 & 14855.41 & 0.1272 & $2.44 \times 10^{14}$ & 0.8346 \\
\hline 0 & $2 s_{1 / 2} 5 d_{5 / 2}$ & 2 & 1 & $2 s_{1 / 2} 2 p_{3 / 2}$ & 1 & 14840.31 & 0.1254 & $2.40 \times 10^{14}$ & 0.8354 \\
\hline 1 & $2 p_{1 / 2} 5 d_{5 / 2}$ & 3 & 0 & $2 p_{1 / 2} 2 p_{3 / 2}$ & 2 & 14837.38 & 0.1955 & $2.67 \times 10^{14}$ & 0.8356 \\
\hline 0 & $2 p_{3 / 2} 4 p_{1 / 2}$ & 2 & 1 & $2 s_{1 / 2} 2 p_{3 / 2}$ & 2 & 14819.28 & 0.0795 & $1.51 \times 10^{14}$ & 0.8366 \\
\hline 0 & $2 p_{3 / 2} 4 p_{1 / 2}$ & 1 & 1 & $2 s_{1 / 2} 2 p_{3 / 2}$ & 2 & 14818.92 & 0.0780 & $2.48 \times 10^{14}$ & 0.8366 \\
\hline 1 & $2 p_{3 / 2} 5 d_{5 / 2}$ & 1 & 0 & $2 p_{3 / 2}^{2}$ & 0 & 14800.25 & 0.0867 & $2.75 \times 10^{14}$ & 0.8377 \\
\hline 0 & $2 s_{1 / 2} 5 d_{3 / 2}$ & 1 & 1 & $2 s_{1 / 2} 2 p_{1 / 2}$ & 0 & 14785.87 & 0.1200 & $3.79 \times 10^{14}$ & 0.8385 \\
\hline 1 & $2 s_{1 / 2} 5 f_{5 / 2}$ & 0 & 1 & $2 s_{1 / 2} 2 p_{1 / 2}$ & 1 & 14774.18 & 0.0309 & $2.92 \times 10^{14}$ & 0.8392 \\
\hline 1 & $2 s_{1 / 2} 5 p_{1 / 2}$ & 1 & 0 & $2 s_{1 / 2}^{2}$ & 0 & 14772.09 & 0.0621 & $1.96 \times 10^{14}$ & 0.8393 \\
\hline 0 & $2 s_{1 / 2} 5 d_{5 / 2}$ & 2 & 1 & $2 s_{1 / 2} 2 p_{1 / 2}$ & 1 & 14763.83 & 0.2993 & $5.66 \times 10^{14}$ & 0.8398 \\
\hline 0 & $2 s_{1 / 2} 5 d_{3 / 2}$ & 1 & 1 & $2 s_{1 / 2} 2 p_{1 / 2}$ & 1 & 14751.86 & 0.1234 & $3.88 \times 10^{14}$ & 0.8404 \\
\hline 0 & $2 p_{3 / 2} 5 p_{1 / 2}$ & 0 & 0 & $2 p_{1 / 2} 2 p_{3 / 2}$ & 1 & 14750.18 & 0.0602 & $5.69 \times 10^{14}$ & 0.8405 \\
\hline
\end{tabular}


TABLE I (cont.)

\begin{tabular}{|c|c|c|c|c|c|c|c|c|c|}
\hline$P_{\mathrm{up}}$ & Upper state & $J_{\text {up }}$ & $P_{\text {low }}$ & Lower state & $J_{\text {low }}$ & $\Delta E[\mathrm{eV}]$ & $g f_{i j}$ & $A_{r}\left[\mathrm{~s}^{-1}\right]$ & $\lambda[\AA]$ \\
\hline 0 & $2 p_{3 / 2} 5 p_{1 / 2}$ & 1 & 0 & $2 p_{1 / 2} 2 p_{3 / 2}$ & 1 & 14748.24 & 0.1497 & $4.71 \times 10^{14}$ & 0.8406 \\
\hline 1 & $2 p_{1 / 2} 5 g_{9 / 2}$ & 2 & 0 & $2 p_{1 / 2} 2 p_{3 / 2}$ & 1 & 14743.74 & 0.1498 & $2.83 \times 10^{14}$ & 0.8409 \\
\hline 1 & $2 p_{3 / 2} 5 s_{1 / 2}$ & 3 & 0 & $2 p_{1 / 2} 2 p_{3 / 2}$ & 2 & 14728.42 & 0.4156 & $5.59 \times 10^{14}$ & 0.8418 \\
\hline 0 & $2 p_{1 / 2} 5 f_{7 / 2}$ & 2 & 1 & $2 s_{1 / 2} 2 p_{3 / 2}$ & 1 & 14727.89 & 0.0780 & $1.47 \times 10^{14}$ & 0.8418 \\
\hline 1 & $2 p_{1 / 2} 5 g_{9 / 2}$ & 2 & 0 & $2 p_{1 / 2} 2 p_{3 / 2}$ & 2 & 14725.30 & 0.1518 & $2.86 \times 10^{14}$ & 0.8420 \\
\hline 0 & $2 p_{1 / 2} 5 p_{1 / 2}$ & 1 & 0 & $2 p_{1 / 2}^{2}$ & 0 & 14683.98 & 0.2391 & $7.46 \times 10^{14}$ & 0.8443 \\
\hline 1 & $2 s_{1 / 2} 5 f_{7 / 2}$ & 3 & 1 & $2 s_{1 / 2} 2 p_{3 / 2}$ & 2 & 13366.01 & 0.5010 & $5.55 \times 10^{14}$ & 0.9276 \\
\hline 1 & $2 p_{3 / 2} 5 d_{3 / 2}$ & 3 & 0 & $2 p_{3 / 2}^{2}$ & 2 & 13307.74 & 0.8216 & $9.02 \times 10^{14}$ & 0.9316 \\
\hline 0 & $2 p_{3 / 2} 5 p_{3 / 2}$ & 2 & 0 & $2 p_{3 / 2}^{2}$ & 2 & 13301.93 & 0.2618 & $4.02 \times 10^{14}$ & 0.9320 \\
\hline 0 & $2 p_{1 / 2} 5 p_{3 / 2}$ & 2 & 0 & $2 p_{1 / 2} 2 p_{3 / 2}$ & 1 & 13294.93 & 0.3248 & $4.98 \times 10^{14}$ & 0.9325 \\
\hline 0 & $2 s_{1 / 2} 5 g_{7 / 2}$ & 2 & 1 & $2 s_{1 / 2} 2 p_{3 / 2}$ & 1 & 13280.24 & 0.3248 & $4.97 \times 10^{14}$ & 0.9336 \\
\hline 0 & $2 p_{1 / 2} 5 d_{3 / 2}$ & 3 & 0 & $2 p_{1 / 2} 2 p_{3 / 2}$ & 2 & 13277.41 & 0.4973 & $5.43 \times 10^{14}$ & 0.9338 \\
\hline 1 & $2 p_{3 / 2} 5 d_{3 / 2}$ & 1 & 0 & $2 p_{3 / 2}^{2}$ & 0 & 13243.94 & 0.2214 & $5.62 \times 10^{14}$ & 0.9361 \\
\hline \multirow[t]{2}{*}{1} & $2 p_{1 / 2} 3 d_{3 / 2}$ & 1 & 0 & $2 s_{1 / 2}^{2}$ & 0 & 11754.18 & 0.0134 & $2.68 \times 10^{13}$ & 1.0548 \\
\hline & & & & & & & $0.0136^{a}$ & $2.70 \times 10^{13 a}$ & $1.0549^{a}$ \\
\hline 0 & $2 p_{3 / 2} 3 p_{3 / 2}$ & 2 & 1 & $2 s_{1 / 2} 2 p_{3 / 2}$ & 2 & 11569.94 & 0.2058 & $2.39 \times 10^{14}$ & 1.0716 \\
\hline 0 & $2 p_{3 / 2} 3 p_{3 / 2}$ & 3 & 1 & $2 s_{1 / 2} 2 p_{3 / 2}$ & 2 & 11537.07 & 0.5814 & $4.80 \times 10^{14}$ & 1.0746 \\
\hline 0 & $2 p_{1 / 2} 3 p_{3 / 2}$ & 1 & 1 & $2 s_{1 / 2} 2 p_{1 / 2}$ & 0 & 11536.07 & 0.1516 & $2.92 \times 10^{14}$ & 1.0747 \\
\hline \multirow[t]{2}{*}{1} & $2 s_{1 / 2} 3 p_{3 / 2}$ & 1 & 0 & $2 s_{1 / 2}^{2}$ & 0 & 11510.65 & 0.3462 & $6.63 \times 10^{14}$ & 1.0771 \\
\hline & & & & & & & $0.3438^{a}$ & $6.59 \times 10^{14 a}$ & $1.0772^{a}$ \\
\hline 0 & $2 p_{3 / 2} 3 p_{3 / 2}$ & 0 & 1 & $2 s_{1 / 2} 2 p_{3 / 2}$ & 1 & 11508.24 & 0.0838 & $4.82 \times 10^{14}$ & 1.0773 \\
\hline 0 & $2 p_{1 / 2} 3 p_{3 / 2}$ & 2 & 1 & $2 s_{1 / 2} 2 p_{1 / 2}$ & 1 & 11507.07 & 0.3672 & $4.22 \times 10^{14}$ & 1.0774 \\
\hline 0 & $2 p_{1 / 2} 3 p_{3 / 2}$ & 1 & 1 & $2 s_{1 / 2} 2 p_{1 / 2}$ & 1 & 11502.17 & 0.0960 & $1.84 \times 10^{14}$ & 1.0779 \\
\hline 0 & $2 p_{3 / 2} 3 p_{3 / 2}$ & 2 & 1 & $2 s_{1 / 2} 2 p_{3 / 2}$ & 1 & 11478.92 & 0.2313 & $2.64 \times 10^{14}$ & 1.0801 \\
\hline 0 & $2 p_{3 / 2} 3 p_{3 / 2}$ & 1 & 1 & $2 s_{1 / 2} 2 p_{3 / 2}$ & 1 & 11447.03 & 0.2206 & $4.18 \times 10^{14}$ & 1.0831 \\
\hline 0 & $2 s_{1 / 2} 3 \mathrm{~d}_{3 / 2}$ & 1 & 1 & $2 s_{1 / 2} 2 p_{1 / 2}$ & 0 & 11364.69 & 0.5569 & $1.04 \times 10^{15}$ & 1.0909 \\
\hline 0 & $2 s_{1 / 2} 3 d_{3 / 2}$ & 2 & 1 & $2 s_{1 / 2} 2 p_{1 / 2}$ & 1 & 11336.26 & 1.4584 & $1.63 \times 10^{15}$ & 1.0937 \\
\hline 1 & $2 p_{3 / 2} 3 d_{3 / 2}$ & 0 & 0 & $2 p_{1 / 2} 2 p_{3 / 2}$ & 1 & 11330.92 & 0.2854 & $1.59 \times 10^{15}$ & 1.0942 \\
\hline 0 & $2 s_{1 / 2} 3 d_{3 / 2}$ & 1 & 1 & $2 s_{1 / 2} 2 p_{1 / 2}$ & 1 & 11330.79 & 0.2599 & $4.83 \times 10^{14}$ & 1.0942 \\
\hline 1 & $2 p_{3 / 2} 3 d_{3 / 2}$ & 1 & 0 & $2 p_{1 / 2} 2 p_{3 / 2}$ & 1 & 11326.26 & 0.7085 & $1.31 \times 10^{15}$ & 1.0946 \\
\hline 1 & $2 p_{3 / 2} 3 d_{3 / 2}$ & 2 & 0 & $2 p_{1 / 2} 2 p_{3 / 2}$ & 1 & 11313.19 & 0.7171 & $7.97 \times 10^{14}$ & 1.0959 \\
\hline 1 & $2 p_{3 / 2} 3 d_{3 / 2}$ & 1 & 0 & $2 p_{1 / 2} 2 p_{3 / 2}$ & 2 & 11308.01 & 0.1492 & $2.76 \times 10^{14}$ & 1.0964 \\
\hline 1 & $2 p_{3 / 2} 3 d_{3 / 2}$ & 3 & 0 & $2 p_{1 / 2} 2 p_{3 / 2}$ & 2 & 11303.74 & 2.0185 & $1.60 \times 10^{15}$ & 1.0968 \\
\hline 1 & $2 p_{3 / 2} 3 d_{3 / 2}$ & 2 & 0 & $2 p_{1 / 2} 2 p_{3 / 2}$ & 2 & 11294.94 & 0.7214 & $7.99 \times 10^{14}$ & 1.0977 \\
\hline 1 & $2 p_{1 / 2} 3 d_{3 / 2}$ & 1 & 0 & $2 p_{1 / 2}^{2}$ & 0 & 11267.96 & 1.1310 & $2.08 \times 10^{15}$ & 1.1003 \\
\hline 0 & $2 p_{3 / 2} 3 p_{1 / 2}$ & 2 & 1 & $2 s_{1 / 2} 2 p_{3 / 2}$ & 2 & 11117.85 & 0.3343 & $3.59 \times 10^{14}$ & 1.1151 \\
\hline 0 & $2 p_{3 / 2} 3 p_{1 / 2}$ & 1 & 1 & $2 s_{1 / 2} 2 p_{3 / 2}$ & 2 & 11116.34 & 0.3274 & $5.85 \times 10^{14}$ & 1.1153 \\
\hline 0 & $2 p_{1 / 2} 3 p_{1 / 2}$ & 0 & 1 & $2 s_{1 / 2} 2 p_{1 / 2}$ & 1 & 11097.78 & 0.1375 & $7.35 \times 10^{14}$ & 1.1172 \\
\hline 0 & $2 p_{1 / 2} 3 p_{1 / 2}$ & 1 & 1 & $2 s_{1 / 2} 2 p_{1 / 2}$ & 0 & 11075.62 & 0.1683 & $2.99 \times 10^{14}$ & 1.1194 \\
\hline \multirow[t]{2}{*}{1} & $2 s_{1 / 2} 3 p_{1 / 2}$ & 1 & 0 & $2 s_{1 / 2}^{2}$ & 0 & 11065.51 & 0.2662 & $4.71 \times 10^{14}$ & 1.1204 \\
\hline & & & & & & & $0.2660^{a}$ & $4.73 \times 10^{14 a}$ & $1.1250^{a}$ \\
\hline 0 & $2 p_{1 / 2} 3 p_{1 / 2}$ & 1 & 1 & $2 s_{1 / 2} 2 p_{1 / 2}$ & 1 & 11041.72 & 0.2984 & $5.26 \times 10^{14}$ & 1.1228 \\
\hline 0 & $2 p_{3 / 2} 3 p_{1 / 2}$ & 2 & 1 & $2 s_{1 / 2} 2 p_{3 / 2}$ & 1 & 11026.83 & 0.3376 & $3.56 \times 10^{14}$ & 1.1243 \\
\hline 0 & $2 s_{1 / 2} 3 d_{5 / 2}$ & 3 & 1 & $2 s_{1 / 2} 2 p_{3 / 2}$ & 2 & 10002.39 & 2.7367 & $1.70 \times 10^{15}$ & 1.2395 \\
\hline 1 & $2 p_{3 / 2} 3 d_{5 / 2}$ & 1 & 0 & $2 p_{3 / 2}^{2}$ & 2 & 9973.29 & 0.1058 & $1.52 \times 10^{14}$ & 1.2431 \\
\hline 0 & $2 p_{1 / 2} 3 p_{3 / 2}$ & 2 & 1 & $2 s_{1 / 2} 2 p_{3 / 2}$ & 1 & 9969.43 & 0.1806 & $1.56 \times 10^{14}$ & 1.2436 \\
\hline 1 & $2 p_{3 / 2} 3 d_{5 / 2}$ & 3 & 0 & $2 p_{3 / 2}^{2}$ & 2 & 9958.99 & 4.4856 & $2.76 \times 10^{15}$ & 1.2449 \\
\hline 1 & $2 p_{3 / 2} 3 d_{5 / 2}$ & 2 & 0 & $2 p_{3 / 2}^{2}$ & 2 & 9941.11 & 1.4350 & $1.23 \times 10^{15}$ & 1.2471 \\
\hline
\end{tabular}


TABLE I (cont.)

\begin{tabular}{c|l|c|c|l|c|c|c|c|c}
\hline \hline$P_{\text {up }}$ & Upper state & $J_{\text {up }}$ & $P_{\text {low }}$ & Lower state & $J_{\text {low }}$ & $\Delta E[\mathrm{eV}]$ & $g f_{i j}$ & $A_{r}\left[\mathrm{~s}^{-1}\right]$ & $\lambda[\AA]$ \\
\hline 1 & $2 p_{1 / 2} 3 d_{5 / 2}$ & 2 & 0 & $2 p_{1 / 2} 2 p_{3 / 2}$ & 1 & 9940.15 & 1.7506 & $1.50 \times 10^{15}$ & 1.2473 \\
0 & $2 s_{1 / 2} 3 d_{5 / 2}$ & 2 & 1 & $2 s_{1 / 2} 2 p_{3 / 2}$ & 1 & 9925.17 & 1.5954 & $1.36 \times 10^{15}$ & 1.2491 \\
1 & $2 p_{1 / 2} 3 d_{5 / 2}$ & 3 & 0 & $2 p_{1 / 2} 2 p_{3 / 2}$ & 2 & 9924.26 & 2.6453 & $1.62 \times 10^{15}$ & 1.2493 \\
1 & $2 p_{1 / 2} 3 d_{5 / 2}$ & 2 & 0 & $2 p_{1 / 2} 2 p_{3 / 2}$ & 2 & 9921.90 & 0.2022 & $1.73 \times 10^{14}$ & 1.2496 \\
1 & $2 p_{3 / 2} 3 d_{5 / 2}$ & 1 & 0 & $2 p_{3 / 2}^{2}$ & 0 & 9904.21 & 1.1995 & $1.70 \times 10^{15}$ & 1.2518 \\
0 & $2 s_{1 / 2} 3 d_{3 / 2}$ & 2 & 1 & $2 s_{1 / 2} 2 p_{3 / 2}$ & 2 & 9889.64 & 0.3500 & $2.97 \times 10^{14}$ & 1.2536 \\
1 & $2 p_{3 / 2} 3 d_{3 / 2}$ & 1 & 0 & $2 p_{3 / 2}^{2}$ & 2 & 9832.95 & 0.2147 & $3.00 \times 10^{14}$ & 1.2609 \\
1 & $2 p_{1 / 2} 3 d_{3 / 2}$ & 1 & 0 & $2 p_{1 / 2} 2 p_{3 / 2}$ & 1 & 9831.56 & 0.1691 & $2.36 \times 10^{14}$ & 1.2610 \\
0 & $2 s_{1 / 2} 3 d_{3 / 2}$ & 1 & 1 & $2 s_{1 / 2} 2 p_{3 / 2}$ & 1 & 9793.16 & 0.1509 & $2.09 \times 10^{14}$ & 1.2660 \\
1 & $2 p_{1 / 2} 3 d_{3 / 2}$ & 2 & 0 & $2 p_{1 / 2} 2 p_{3 / 2}$ & 2 & 9785.75 & 0.2975 & $2.47 \times 10^{14}$ & 1.2669 \\
1 & $2 p_{3 / 2} 3 d_{3 / 2}$ & 1 & 0 & $2 p_{3 / 2}^{2}$ & 0 & 9763.87 & 0.1066 & $1.47 \times 10^{14}$ & 1.2698 \\
0 & $2 s_{1 / 2} 3 s_{1 / 2}$ & 1 & 1 & $2 s_{1 / 2} 2 p_{3 / 2}$ & 2 & 9331.87 & 0.1628 & $2.05 \times 10^{14}$ & 1.3286 \\
1 & $2 p_{3 / 2} 3 s_{1 / 2}$ & 1 & 0 & $2 p_{3 / 2}^{2}$ & 2 & 9291.87 & 0.1634 & $2.04 \times 10^{14}$ & 1.3343 \\
1 & $2 p_{1 / 2} 3 s_{1 / 2}$ & 0 & 0 & $2 p_{1 / 2} 2 p_{3 / 2}$ & 1 & 9276.20 & 0.0651 & $2.43 \times 10^{14}$ & 1.3365 \\
0 & $2 s_{1 / 2} 3 s_{1 / 2}$ & 0 & 1 & $2 s_{1 / 2} 2 p_{3 / 2}$ & 1 & 9275.54 & 0.0602 & $2.25 \times 10^{14}$ & 1.3366 \\
1 & $2 p_{1 / 2} 3 s_{1 / 2}$ & 1 & 0 & $2 p_{1 / 2} 2 p_{3 / 2}$ & 2 & 9260.04 & 0.1575 & $1.95 \times 10^{14}$ & 1.3389 \\
\hline
\end{tabular}

${ }^{a}$ Retrieved from [26]

The $n p-2 p$ dominates the magnetic dipole M1 transitions. The strongest transition is identified to be $3 p_{1 / 2}-2 p_{3 / 2}$, with radiative transition probability of $6.1892 \times 10^{+10} \mathrm{~s}^{-1}$ at $\lambda=1.3302 \AA$. In [31], the strongest transitions of M1 atomic data are collected in Table SIII. The other M1 transition groups are $n s-2 s, n f-2 p$ and $n d-2 s$. The intensity ratio of M1 transitions compared to E1 transitions (M1/E1) is about $10^{-5}$.

Magnetic quadrupole M2 transitions are divided into five groups: $n p-2 p$, which is the dominant group, $n p-2 s, n s-2 p, 2 s-n f$ and $n g-2 p$. The strongest transition is $6 p_{3 / 2}-2 p_{1 / 2}$, with radiative transition probability of $1.1323 \times 10^{+11} \mathrm{~s}^{-1}$ at $\lambda=0.6560 \AA$. The intensity ratio of M2 transitions compared to E1 transitions (M2/E1) is about $10^{-5}$. Atomic parameters of the most important transitions are listed in Table SIV in [31].

Finally, magnetic-octupole M3 transitions are divided into five groups: $n d-2 s, n f-2 p, n p-2 p, n h-2 p$ and $n g-2 s$. The $n f-2 p$ group has the strongest transition $4 f_{7 / 2}-2 p_{1 / 2}$, with radiative transition probability of $4.1869 \times 10^{+8} \mathrm{~s}^{-1}$ at $\lambda=0.8401 \AA$. The transition lines of M3 are listed for the strongest lines in Table SV found in the supplementary material [31]. The intensity ratio of M3 transitions compared to E1 transitions (M3/E1) is about $10^{-7}$.

\section{Theoretical spectra}

Synthetic spectra are constructed for the electric and magnetic multipole transitions (E1, E2, E3, M1, M2 and M3) by using the Doppler line profile with low energy. Line intensity is normalized to unity in arbitrary units. These spectra are produced in order to simulate the spectra of low-density tungsten plasma (electron density $n_{e} \leq 10^{13} \mathrm{~cm}^{-1}$ ), where most ions are in ground states and electrons excitation and de-excitation are the main processes that give rise to a real spectrum. Constructed synthetic spectra for low-density plasma are helpful in the identification of spectral features and in the diagnostic of hot dense plasma, whose synthetic spectra should be calculated by collisional-radiative models. Using the collisional radiative model is the best way to produce a theoretical spectrum for experimental plasma diagnostics which involves many experimental parameters. This is not the case in this study. However, the locations of the transitions (wavelengths) do not change in the theoretical spectrum regardless of whether a simplified model or the collisional radiative model is used.

Theoretical spectra of allowed and forbidden transitions are shown in layers in Fig. 1. The plot is for the wavelengths $\lambda[\AA]$ versus the relative intensities (Rel. Int.) in arbitrary units [a.u.]. The lower layer is for E1 transitions and the layers are in the sequence for M1, E2, M2, E3 and M3 transitions. It is evident that the spectral ranges of these transitions are comparable. The E1 spectrum has many strong E2 lines. The spectra of forbidden transitions have less transition lines. These synthetic spectra are "finger-prints" for the structure of the Be-like tungsten ion. Locations of transition lines remain unchanged in the spectra of hot dense plasma but lines intensities are subject to change due to contributions of other electronic processes.

It is found that allowed transitions of the Be-like tungsten ion are located in the spectral range of $0.7-1.4 \AA$. The spectrum of M1 has the spectral 

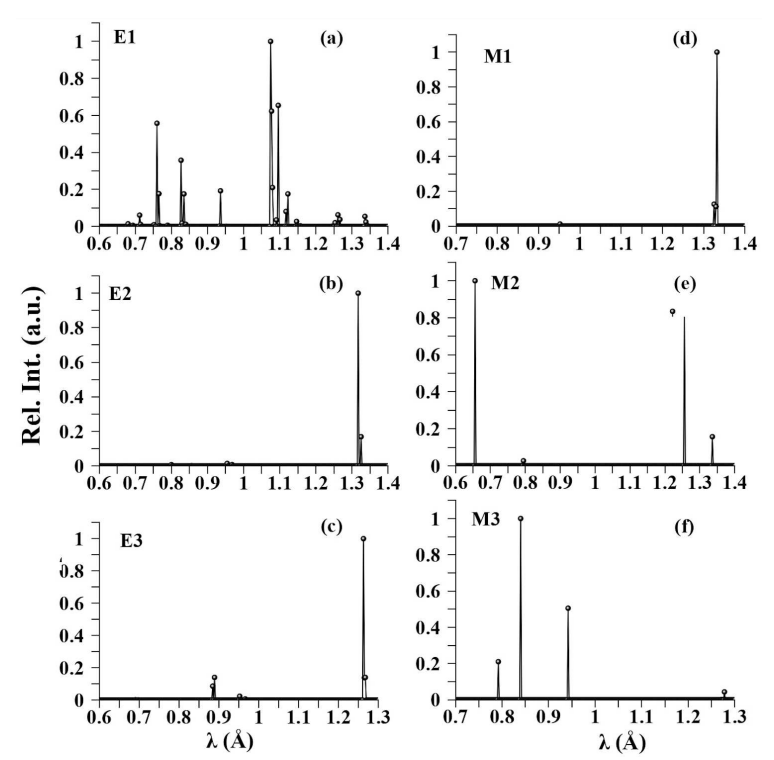

Fig. 1. Theoretical spectrum of multipole transitions of Be-like W: (a) E1 transitions, (b) E2 transitions, (c) E3 transitions, (d) M1 transitions, (e) M2 transitions, (f) M3 transitions.

range of $0.8-1.4 \AA$ (the second layer of Fig. 1). The spectral range of E2 is $0.9-1.4 \AA$. Further, the spectrum of M2 is in the spectral range of $0.65-$ $1.4 \AA$. The transition lines of E3 spectrum are in the spectral range of $0.7-1.3 \AA$. Finally, the last layer for M3 transitions is in the spectral range of $0.75-1.3 \AA$.

\section{Summary and conclusions}

The relativistic configuration interaction method of the FAC is applied to produce atomic data for multipole transitions of the Be-like tungsten ion. Wavelengths, radiative transition rates and oscillator strength values for electric-dipole E1, electricquadrupole E2, electric-octupole E3, magneticdipole M1, magnetic-quadrupole M2 and magneticoctupole M3 are calculated, tabulated and discussed. The calculated energies of the multipole transition lines are in the $\mathrm{X}$-ray region, where wavelengths are found to be in the range of $0.6-1.4 \AA$. Several blending instances among transition lines of multipole transitions are detected. The comparison with the available published data calculated by GRASP2K shows good agreement which indicates that the atomic data calculated in this paper for spectral parameters are accurate enough to be used in diagnosing and modeling of tungsten plasma.

\section{References}

[1] G.A. Wurden, B.J. Peterson, Rev. Sci. Instrum. 70, 255 (1999).

[2] S. Cowell, A. Hayes, Nuclear Weapons Program Highlights T16, 106 (2006).
[3] K. Asmussen, K.B. Fournier, J.M. Laming, R. Neu, J.F. Seely, R. Dux, W. Engelhardt, J.C. Fuchs, and ASDEX Upgrade Team, Nucl. Fusion 38, 967 (1998).

[4] T. Putterich, R. Neu, C. Biedermann, R. Radtkeand, and ASDEX Upgrade Team, J. Phys. B 38, 3071 (2005).

[5] M. Klapisch, J.L. Schwob, B.S. Fraenkel, J. Oreg, J. Opt. Soc. Am. 67, 148 (1977).

[6] J. Clementson, T. Lennartsson, P. Beiersdorfer, Atoms 3, 407 (2015).

[7] R. Neu, K.B. Fournier, D. Schlogl, J. Rice, J. Phys. B 30, 5065 (1997).

[8] A. Shlyaptseva, D. Fedin, S. Hamasha, C. Harris, V. Kantsyrev, P. Neill, N. Ouart, U.I. Safronova, Rev. Sci. Instrum. 75, 3750 (2004).

[9] S.R. Elliott, P. Beiersdorfer, B.J. MacGowan, J. Nilsen, Phys. Rev. A 52, 2689 (1995).

[10] A.S. Shlyaptseva, D.A. Fedin, S.M. Hamasha, S.B. Hansen, C. Harris, V.L. Kantsyrev, P. Neill, N. Ouart, Rev. Sci. Instrum. 74, 1947 (2003).

[11] K.B. Fournier, At. Data Nucl. Data Tables 68, 1 (1998).

[12] M. Klapisch, P. Mandelbaum, A. Barshalom, J.L. Schwob, J. Opt. Soc. Am. 71, 1276 (1981).

[13] P. Beiersdorfer, J.A. Britten, G.V. Brown, Phys. Scr. T92, 268 (2001).

[14] R. Radtke, C. Biedermann, J.L. Schwob, P. Mandelbaum, R. Doron, Phys. Rev. A 64, 012720 (2001).

[15] S.B. Utter, P. Beiersdorfer, E. Trabert, Can. J. Phys. 80, 1503 (2002).

[16] P. Neill, C. Harris, A.S. Safronova, S. Hamasha, S. Hansen, U.I. Safronova, P. Beiersdorfer, Can. J. Phys. 82, 931 (2004).

[17] Yu. Ralchenko, J. Reader, J.M. Pomeroy, J.N. Tan, J.D. Gillaspy, J. Phys. B 40, 3891 (2007).

[18] A. Bar-Shalom, M. Klapisch, Comput. Phys. Commun. 50, 375 (1988).

[19] M.F. Gu, Can. J. Phys. 86, 675 (2008).

[20] L. Özdemir, G. Gunday Konan, S. Kabakci, Acta Phys. Pol. A 124, 649 (2013.

[21] S. Aggarwal, A.K.S. Jha, M. Mohan, Can. J. Phys. 91, 394 (2013).

[22] S. Aggarwal, Chin. Phys. B 23, 093203 (2014).

[23] Dipti, T. Das, L. Sharma, R. Srivastava, R. Swed. Acad. Sci. 89, 085403 (2014). 
[24] Y. Ralchenko, J.N. Tan, J.D. Gillaspy, J.M. Pomeroy, E. Silver, Phys. Rev. A 74, 042514 (2006).

[25] Y.J. Rhee, D.H. Kwon, Int. J. Mass Spectrom. 271, 45 (2008).

[26] N. Singh, S. Aggarwal, M. Mohan, J. Electron. Spectrosc. 229, 124 (2018).

[27] E. Biémont, P. Quinet, A.Y. Faenov, I. Skobelev, J. Nilsen, V.M. Romanova, M. Scholz, L. Karpinski, A. Szydlowski, Phys. Scr. 61, 555 (2000).
[28] A.J. Smith, P. Beiersdorfer, V. Decaux, K. Widmann, A. Osterheld, M. Chen, Phys. Rev. A 51, 2808 (1995).

[29] P. Jönsson, G. Gaigalas, J. Bieroń, C. Froese Fischer, I.P. Grant, Comput. Phys. Commun. 184, 2197 (2013).

[30] S. Eser, L. Ozdemir, Acta Phys. Pol. A 133, 1324 (2018).

[31] K. Hamasha, Acta Phys. Pol. A 139, 151.S1 (2021), The suppl. material with Tables SI-SV, available online only. 